\title{
Analytical description of the 1s exciton linewidth temperature dependence in transition metal dichalcogenides
}

Henriques, J. C.G.; Mortensen, N. A.; Peres, N. M.R.

Published in:

Physical Review B

Link to article, DOI:

10.1103/PhysRevB.103.235402

Publication date:

2021

Document Version

Publisher's PDF, also known as Version of record

Link back to DTU Orbit

Citation (APA):

Henriques, J. C. G., Mortensen, N. A., \& Peres, N. M. R. (2021). Analytical description of the 1s exciton linewidth temperature dependence in transition metal dichalcogenides. Physical Review B, 103(23), [235402].

https://doi.org/10.1103/PhysRevB.103.235402

\section{General rights}

Copyright and moral rights for the publications made accessible in the public portal are retained by the authors and/or other copyright owners and it is a condition of accessing publications that users recognise and abide by the legal requirements associated with these rights.

- Users may download and print one copy of any publication from the public portal for the purpose of private study or research.

- You may not further distribute the material or use it for any profit-making activity or commercial gain

- You may freely distribute the URL identifying the publication in the public portal 


\title{
Analytical description of the $1 s$ exciton linewidth temperature dependence in transition metal dichalcogenides
}

\author{
J. C. G. Henriques $\odot,^{1,2,3}$ N. A. Mortensen $\odot,{ }^{3,4,5}$ and N. M. R. Peres $\oplus^{1,2, *}$ \\ ${ }^{1}$ Department and Centre of Physics, and QuantaLab, University of Minho, Campus of Gualtar, 4710-057 Braga, Portugal \\ ${ }^{2}$ International Iberian Nanotechnology Laboratory (INL), Avenida Mestre José Veiga, 4715-330 Braga, Portugal \\ ${ }^{3}$ Center for Nano Optics, University of Southern Denmark, Campusvej 55, DK-5230 Odense M, Denmark \\ ${ }^{4}$ Danish Institute for Advanced Study, University of Southern Denmark, Campusvej 55, DK-5230 Odense M, Denmark \\ ${ }^{5}$ Center for Nanostructured Graphene, Technical University of Denmark, DK-2800 Kongens Lyngby, Denmark
}

(Received 25 March 2021; revised 20 May 2021; accepted 24 May 2021; published 1 June 2021)

\begin{abstract}
We obtain an analytical expression for the linewidth of the $1 \mathrm{~s}$ exciton as a function of temperature in transition metal dichalcogenides. The total linewidth, as a function of temperature, is dominated by three contributions: (i) the radiative decay (essentially temperature independent), (ii) the phonon-induced intravalley scattering, and (iii) the phonon-induced intervalley scattering. Our approach uses a variational Ansatz to solve the Wannier equation, allowing for an analytical treatment of the excitonic problem, including rates of the decay dynamics. Our results are in good agreement with experimental data already present in the literature and can be used to readily predict the value of the total linewidth at any temperature in the broad class of excitonic two-dimensional materials.
\end{abstract}

DOI: 10.1103/PhysRevB.103.235402

\section{INTRODUCTION}

The optical response of transition metal dichalcogenides (TMDs) is characterized by strong absorption peaks due to excitons formed at the $\mathrm{K}$ and $\mathrm{K}^{\prime}$ points of the Brillouin zone $[1,2]$. The peaks commonly seen in absorption and photoluminescence spectra are associated with the so-called bright excitons, particularly to the $s$ states (with vanishing angular momentum) of the $A$ and $B$ series [1,3]. The existence of two distinct series of resonances comes about because of the strong spin-orbit coupling which breaks the spin degeneracy in these systems [4]. The effect of spin-orbit coupling is particularly noticeable in the valence band, where in the $\mathrm{K}$ valley, the band with positive spin polarization is shifted downward relative to the band with opposite spin. In the conduction band, this effect is barely perceptible in $\mathrm{MoS}_{2}$ but plays a significant role in $\mathrm{WS}_{2}$ and $\mathrm{WSe}_{2}$ [5].

In addition to the optically active exciton states, TMDs present a plethora of dark excitons [6-8], corresponding to exciton states which may be formed but cannot be directly accessed optically. Examples of these are the $p$ states [9], the spin-opposed excitons [10], and the momentum dark excitons [11]. The first ones cannot be directly activated with optical excitation due to angular momentum conservation; however, they can be accessed in a pump-probe setup where the pump laser populates the $1 s$ exciton state, and the probe induces transitions between the $1 s$ and the $p$ states [12]. The spinopposed excitons are composed of an electron and a hole with opposite spins and are not optically accessible since the electron-light interaction does not produce the required spin flip. At last, the momentum dark states are composed of an electron and a hole with equal spin but located in different momentum points of the Brillouin zone. Due to the small

\footnotetext{
*peres@ fisica.uminho.pt
}

momentum carried by photons, these states are not optically active; however, the momentum mismatch may be overcome, and they may be accessed due to additional coupling with phonons [13].

The rich optical properties of TMDs make them some of the most prominent materials in the area of nanodevices, with applications ranging from photodetectors to biosensors and valleytronics [14-18]. The application of TMDs in devices at finite temperature is limited by the linewidth of the $1 s$ excitonic line [19]. Therefore, an understanding of how this linewidth depends both on temperature and on the dielectric media surrounding the two-dimensional (2D) material is key to a complete description of device performance based on this class of materials. The effect of temperature is mostly dominated by carrier-phonon scattering, and understanding the details of how this scattering mechanism affects the linewidth is essential. Even though this process has already been studied both theoretically and experimentally $[7,11]$, a simple analytical framework giving insight into the underlying physics is lacking and is necessary.

In this paper, we derive analytical expressions describing the linewidth of the lowest-lying excitonic resonance in TMDs at finite temperature. We account for the contributions of radiative recombination and exciton-phonon scattering, including both intravalley and intervalley processes. To describe these contributions, we obtain the exciton energies and wave functions from the solution of the Wannier equation using a variational Ansatz. The combination of the methods and concepts in this paper give a unified analytical picture of the phonon linewidth in TMDs.

\section{WANNIER EQUATION}

\section{A. Derivation}

The starting point of our discussion is the introduction of the exciton creation operator. This bosonic operator describes 
the creation of an exciton with the electron in the point $\xi_{e}$ and the hole in the point $\xi_{h}$ of the Brillouin zone, with center of mass momentum $\mathbf{Q}$ (measured relative to the $\xi_{e}$ and $\xi_{h}$ ) and quantum numbers $v$ (containing both the principal and angular quantum numbers). This operator is composed of a superposition of electronic operators that annihilate an electron in the valence band and create one in the conduction band, weighted with the Fourier transform of the exciton wave function $\psi_{\nu}(\mathbf{k})$, that is,

$$
b_{\mathbf{Q}, v}^{\dagger, \xi_{e}, \xi_{h}}=\frac{1}{\sqrt{A}} \sum_{\mathbf{k}} \psi_{\nu, \mathbf{k}}^{\xi_{e} \xi_{h}} c_{\mathbf{k}+\alpha_{\xi_{e}, \xi_{h}}^{\dagger}, \mathbf{Q}, c} c_{\mathbf{k}-\beta_{\xi_{e}, \xi_{h}} \mathbf{Q}, v}^{\xi_{h}}
$$

When this operator acts on the excitonic ground state (a full valence band and an empty conduction band), we find

$$
b_{\mathbf{Q}, v}^{\dagger, \xi_{e}, \xi_{h}}|G S\rangle=\left|\xi_{e}, \xi_{h}, v, \mathbf{Q}\right\rangle .
$$

Then we assume that the Hamiltonian (containing kinetic and potential energy contributions) is diagonal in this operator, with the eigenvalue $E_{v, \mathbf{Q}}=E_{g}+E_{v}+\hbar^{2} \mathbf{Q}^{2} / 2 M$, where $E_{g}$ is the band gap (obtained from, for example, $G W$ calculations accounting for the exchange correction), $E_{v}$ the exciton binding energy, and $\hbar^{2} \mathbf{Q}^{2} / 2 M$ the kinetic energy of the center of mass. Afterward, we compute the commutator of the Hamiltonian with the exciton operator using its representation in terms of exciton operators and in terms of electron operators. Demanding the equality of both results, the Bethe-Salpeter equation (BSE) follows; its solution yields the binding energies and wave functions of the excitonic problem. The interaction potential in the electronic Hamiltonian is treated within the Rytova-Keldysh (RK) formalism [20-22], which crucially accounts for the nonlocal screening in the TMD monolayer. Fourier transforming the BSE (see Appendix A), we find the Wannier equation [23], a differential equation in real space reading

$$
-\frac{\hbar^{2}}{2 \mu_{\xi_{e} \xi_{h}}} \nabla^{2} \psi_{v}(\mathbf{r})+V_{\mathrm{RK}}(\mathbf{r}) \psi_{v}(\mathbf{r})=E_{\nu} \psi_{\nu}(\mathbf{r})
$$

where $\mu_{\xi_{e} \xi_{h}}$ is the reduced mass of the electron-hole pair, with the former and the latter located in the $\xi_{e}$ and $\xi_{h}$ valley, respectively. In Eq. (3), $V_{\mathrm{RK}}(\mathbf{r})$ is the RK potential and $\psi_{\nu}(\mathbf{r})$ the exciton wave function in real space. The RK potential follows from the solution of the Poisson equation for a point charge in a thin dielectric and reads $[20,21]$

$$
V_{\mathrm{RK}}(\mathbf{r})=-\frac{e^{2}}{4 \pi \epsilon_{0}} \frac{\pi}{2 r_{0}}\left[H_{0}\left(\frac{\kappa r}{r_{0}}\right)-Y_{0}\left(\frac{\kappa r}{r_{0}}\right)\right],
$$

where $e$ is the electron charge; $\epsilon_{0}$ the permittivity of vacuum; $\kappa$ is the mean dielectric constant of the media above and below the TMD layer; $r_{0}$ is a material parameter which can be macroscopically associated with a screening length and is microscopically related to the polarizability of the monolayer; and $H_{0}$ is the Struve function and $Y_{0}$ the Bessel function of the second kind, both of order zero. The difference between momentum bright and momentum dark excitons lies solely on the value of the reduced mass, which should be computed with the adequate effective masses of the carriers. To obtain the location of the exciton states, the structure of the electronic bands must also be known. In this paper, we used the band parameters found from ab initio calculations in Ref. [5].
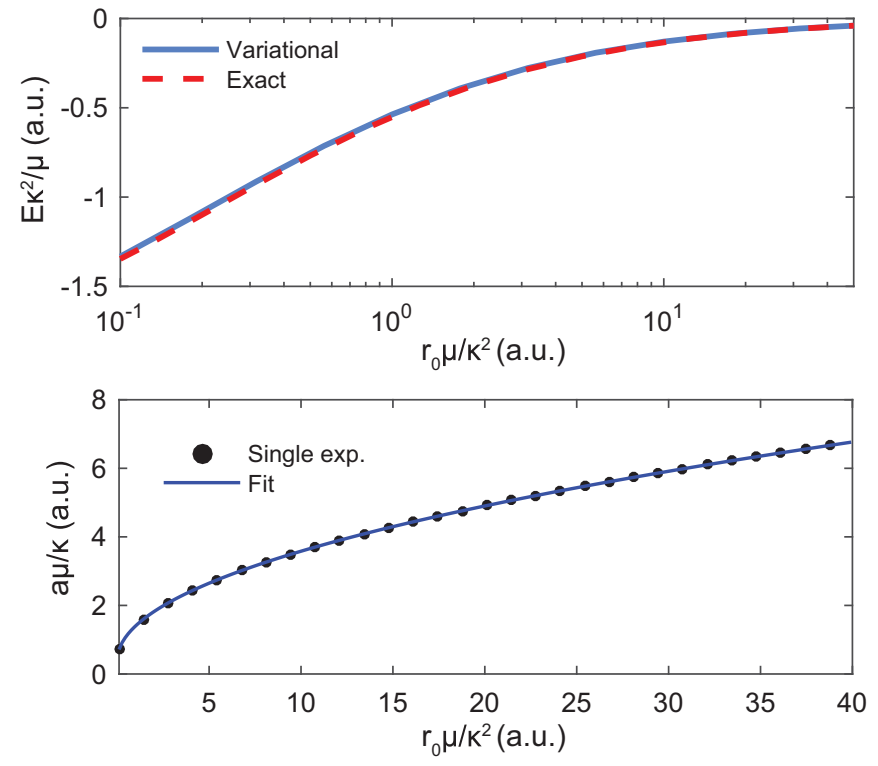

FIG. 1. (Top) Comparison between the values of $\tilde{E}$ obtained using a variational approach and expanding the wave functions in a Gaussian basis and numerically diagonalizing the Hamiltonian. (Bottom) values of $\mu a / \kappa$ obtained with the single exponential Ansatz, as well as the fit to $\chi+b\left(\tilde{r}_{0}\right)^{c}$, which yields Eq. (7). The different quantities depicted in both panels are given in atomic units (a.u.).

We stress that the goal of our approach is not so much the quantitative accuracy, but rather the possibility of obtaining qualitative and physically transparent results using simpler techniques which can be explored analytically.

\section{B. Variational solution}

While the direct solution of the BSE requires a delicate numerical diagonalization, Eq. (3) can be solved with a variational approach [24], which allows us to develop an analytical framework and gain insight into the underlying physics. Different variational Ansätze can be employed to solve this problem; here, we opt to use the simplest one, a single evanescent exponential

$$
\psi_{1 s}(\mathbf{r})=\sqrt{\frac{2}{\pi a^{2}}} e^{-r / a},
$$

like the wave function of the hydrogen atom. The value of $a$ is determined from the minimization of the energy in Eq. (3). Following the scaling procedure to a dimensionless representation proposed in Ref. [25], and working for the moment in atomic units (a.u.), we introduce the following quantities:

$$
\frac{r_{0} \mu}{\kappa^{2}}=\tilde{r}_{0}, \quad \frac{\kappa^{2}}{\mu} E=\tilde{E}, \quad \frac{\mu r}{\kappa}=\rho,
$$

which allow us to conveniently describe the excitonic problem with a single effective parameter $\tilde{r}_{0}=r_{0} \mu / \kappa^{2}$ instead of the three independent ones we currently have. In the top panel of Fig. 1, we plot the value of $\tilde{E}$ vs $\tilde{r}_{0}$ obtained using the variational approach and compare it with the result found by numerically diagonalizing the Hamiltonian. The depicted interval of $\tilde{r}_{0}$ covers the relevant region of the parameter space for the most common TMDs with different dielectric back- 
grounds. The agreement between the variational and the exact results is clear. The differences between the two solutions, corresponding to a few millielectronvolts, are not expected to significantly change the final results. Another possible variational approach uses the more sophisticated Ansatz [25]

$$
\psi(\mathbf{r})=\mathcal{N}\left(e^{-r / \alpha}+\beta e^{-r / \gamma}\right),
$$

with $\mathcal{N}$ a normalization constant and $\alpha, \beta$, and $\gamma$ the variational parameters. Although this double exponential Ansatz yields more accurate results (in fact it produces a near perfect description of the exact results), we do not consider the increased precision to be sufficient to justify the more involved analytical treatment.

Now noting that, in the usual TMDs, one finds $r_{0} \mu=$ $30-40$ atomic units [25,26], and considering $\kappa \in[1,5]$ (a reasonable range of values for typical experiments), we perform a power-law fit of $a$ vs $\tilde{r}_{0}$ in the region of interest of the parameter space. Doing so, we realize that, in International System of Units (SI) units, $a$ is accurately described by

$$
\frac{a}{a_{B}} \simeq \frac{\kappa}{\mu / m_{0}} \chi+\sqrt{\frac{r_{0} / a_{B}}{\mu / m_{0}}},
$$

where $\chi=0.4$ is determined by the fitting process, $m_{0}$ is the bare electron mass, and $a_{B}$ is the Bohr radius. The comparison between Eq. (7) and the values found directly from the variational procedure is depicted in the bottom panel of Fig. 1. If the fit had been performed in a region corresponding to smaller values of $r_{0}$, and consequently smaller $\tilde{r}_{0}$, the value of $\chi$ would become ever closer to 0.5 , just like in the 2D hydrogen atom, and in agreement with the limiting case

$$
\lim _{r_{0} \rightarrow 0} V_{\mathrm{RK}}(r)=V_{\text {Coulomb }}(r) .
$$

The fact that, in the relevant region of parameters for TMDs, the value of $\chi$ differs from 0.5 reflects the nonhydrogenic nature of excitons in these 2D systems; the term $\sqrt{r_{0} / \mu}$ further enhances this difference.

We emphasize that having an analytical description for $a$ is a rather useful achievement since we can now obtain an analytical expression for the binding energies of excitons in the $1 s$ state. In Appendix B, we study in more detail how the binding energies found with the variational method compare with those obtained by numerically solving the Wannier equation.

\section{Exciton energy landscape}

Using the single exponential variational Ansatz, we solve the Wannier equation and obtain the excitonic energy dispersion for the lowest-lying exciton in the vicinity of the principle high-symmetry points of the excitonic Brillouin zone for $\mathrm{MoS}_{2}, \mathrm{MoSe}_{2}, \mathrm{WS}_{2}$, and $\mathrm{WSe}_{2}$ on a substrate of $\mathrm{SiO}_{2}$. The energy spectrum is depicted in Fig. 2 for excitons, where the electron and hole are located in the $\mathrm{K}$ valley (KK exciton), excitons where the hole is in the $\mathrm{K}$ valley, and the electron either in $\Lambda$ or $\mathrm{K}^{\prime}$ ( $\mathrm{K} \Lambda$ and $\mathrm{KK}^{\prime}$ excitons, respectively). The values of the binding energies for each exciton are shown in Appendix B. To obtain these results, we considered the effective masses and relative band edge energies presented in Ref. [5], where these quantities were obtained from density
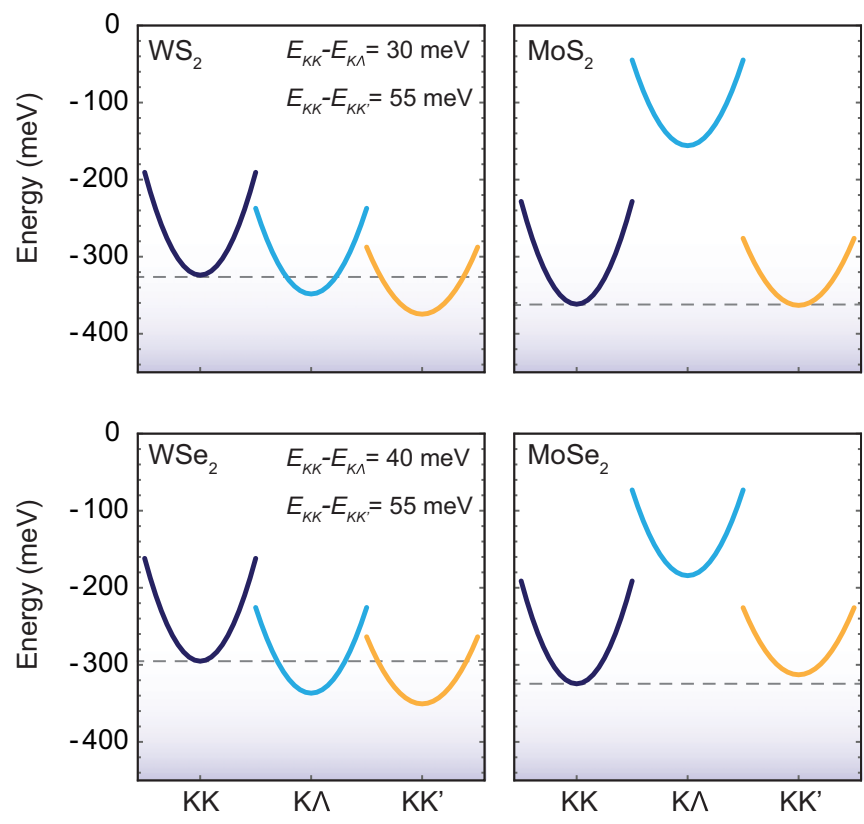

FIG. 2. Exciton band structure in four different transition metal dichalcogenides (TMDs). The excitons are labeled by the hole and electron valley, respectively, e.g., $\mathrm{K} \Lambda$ refers to a momentum dark exciton with the hole in the $\mathrm{K}$ valley and the electron in the $\Lambda$ valley. The energies are measured with respect to the bottom of the conduction band in the $\mathrm{K}$ valley. The effective masses and relative band edge energies of Ref. [5] were used.

functional theory (DFT) calculations using the Perdew-BurkeErnzerhof generalized gradient approximation. The effective masses used for the $\Lambda$ valley were obtained as the square root of the product of the effective masses along the $x$ and $y$ directions.

When the electron and hole are located in the $\mathrm{K}$ valley, the lowest-lying bright exciton is composed of carriers belonging to the spin-down bands (for the four considered TMDs). Since in TMDs the valley and spin degrees of freedom are coupled, in the $\mathrm{K}^{\prime}$ valley, the lowest-lying bright exciton would have carriers with the opposite spin to the ones in the $\mathrm{K}$ valley. Henceforth, we will consider all the relevant carriers to have downward pointing spin because interaction with phonons does not lead to spin flips.

The most important aspect of Fig. 2 is the existence of momentum forbidden excitonic states below the optically active KK exciton in tungsten-based TMDs. Regarding the $\mathrm{K} \Lambda$ exciton, the main difference between $\mathrm{MoX}_{2}$ and $\mathrm{WX}_{2}$ TMDs is the position of the conduction band edge near the $\Lambda$ valley since, for the former, the band edge sits $\sim 200 \mathrm{meV}$ above the bottom of the conduction band in the $\mathrm{K}$ valley, while for the latter, the separation is inferior to $30 \mathrm{meV}$. This small energy separation, combined with the larger effective masses in the $\Lambda$ valley (leading to more tightly bound excitons), is responsible for the appearance of the $\mathrm{K} \Lambda$ excitons below the bright one in tungsten-based TMDs. In MoX $\mathrm{X}_{2}$, the conduction band edge at the $\Lambda$ point is located too far up, and the $\mathrm{K} \Lambda$ excitons are formed above the the bright one.

As far as the $\mathrm{KK}^{\prime}$ exciton is concerned, its position is mainly determined by the effect of spin-orbit coupling in the 
conduction band near the $\mathrm{K}$ and $\mathrm{K}^{\prime}$ valleys. Since in TMDs the spin and valley degrees of freedom are coupled, the position of a band with a given spin polarization in the $\mathrm{K}$ valley coincides with the position of the band with opposite spin in the $\mathrm{K}^{\prime}$ valley. The spin splitting in the conduction band is rather small for TMDs with molybdenum, justifying the similar positions of the KK and $\mathrm{KK}^{\prime}$ excitons in $\mathrm{MoS}_{2}$ and $\mathrm{MoSe}_{2}$ in Fig. 2. For $\mathrm{WS}_{2}$ and $\mathrm{WSe}_{2}$, the spin splitting in the conduction band is larger, leading to an increased difference in the $\mathrm{KK}$ and $\mathrm{KK}^{\prime}$ excitons. Moreover, the spin splittings have opposite signs depending on the transition metal atom; the sign in $\mathrm{WS}_{2}$ and $\mathrm{WSe}_{2}$ further shifts the $\mathrm{KK}^{\prime}$ exciton below the optically bright one.

Comparing our results with the ones found by Malic et al. in Ref. [6], where the binding energies were obtained from the numerical solution of the Wannier equation in momentum space, a good agreement is seen; this emphasizes the merits of the variational approach we apply here. Furthermore, we note that our results are also in line with the ones found from DFT calculations in Ref. [27].

\section{HOMOGENEOUS LINEWIDTH}

Now that the equation governing the excitonic problem was found and a simple variational approach of solving it was introduced, we can move onto the computation of the homogeneous linewidth of the $1 s$ exciton in TMDs.

\section{A. Radiative linewidth}

Starting with the radiative linewidth [28] and using Fermi's golden rule, we write in the dipole approximation

$$
\begin{aligned}
\gamma_{\mathrm{rad}}=\frac{\hbar}{\tau_{1 s}}= & 2 \pi \sum_{\mathbf{q}}\left|\left\langle G S ; 1_{\mathbf{q}}|e \mathbf{E} \cdot \mathbf{r}| 1 s ; \mathbf{Q}=0\right\rangle\right|^{2} \\
& \times \delta\left(E_{g}+E_{1 s}-\hbar \omega_{\mathbf{q}}\right),
\end{aligned}
$$

where $\hbar \omega_{\mathbf{q}}$ is the energy of a photon with momentum $\mathbf{q}$, and the matrix element describes the radiative recombination of an exciton in the state $1 s$ such that, in the final state, there is one extra photon in the light field; $E_{g}$ is the full band gap between the edges of the spin-down bands at the $\mathrm{K}$ valley (obtained from, e.g., $G W$ calculations [5]); and $E_{1 s}$ is the exciton binding energy ( $E_{g}+E_{1 s}$ is the energy of the initial state). Quantizing $\mathbf{E} \cdot \mathbf{r}$ in terms of photon and exciton operators and carrying out the calculation (see Appendix C), one finds

$$
\gamma_{\mathrm{rad}}=\frac{8 \pi}{\kappa} \alpha\left(\hbar v_{F}\right)^{2} \frac{E_{g}+E_{1 s}}{\left(E_{g}^{\tau, s_{z}}\right)^{2}} \psi_{1 s}^{2}(\mathbf{r}=0),
$$

where $\alpha \sim 1 / 137$ is the fine-structure constant, $v_{F}$ is the Fermi velocity (which may be obtained from first-principles calculations of the electronic band structure), $\tau= \pm 1$ and $s_{z}= \pm 1$ are the valley $\left(\mathrm{K} / \mathrm{K}^{\prime}\right)$ and spin (up/down) indices, respectively, and $E_{g}^{\tau, s_{z}}$ is the noninteracting band gap, which is spin and valley dependent and is obtained from first-principles calculations $[5,29]$. Note that $E_{g}$ and $E_{g}^{\tau, s_{z}}$ are not identical since the former contains exchange corrections which widen the band gap. The dependence of $\psi_{1 s}^{2}(\mathbf{r}=0)$ on $\kappa, \mu$, and $r_{0}$ follows from Eq. (7).
Analyzing Eq. (9), we expect the radiative linewidth to increase as the band gap of the material widens and to decrease approximately according to a power law as the surrounding dielectric screening increases. Although our expression is independent of the temperature, the radiative linewidth may vary with it as a result of modifications in the band structure. This, however, is beyond the scope of this paper. For the most common TMDs, i.e., $\mathrm{MoS}_{2}, \mathrm{MoSe}_{2}, \mathrm{WS}_{2}$, and $\mathrm{WSe}_{2}$, we find $\gamma_{\mathrm{rad}} \sim 5 \mathrm{meV}$, in agreement with other independent results $[28,30]$. Furthermore, we note that, although the expression we obtained for the radiative linewidth refers to the $1 s$ state, it can be easily generalized to other states by replacing $E_{1 s}$ and $\psi_{1 s}$ with the appropriate binding energies and wave functions (this easily leads to the result that states whose wave function vanishes at $\mathbf{r}=0$, such as the $p$ states, are optically dark).

\section{B. Phonon-induced linewidth}

To describe the linewidth originating from exciton-phonon coupling, one must consider the mechanisms responsible for phonon-driven carrier scattering. In the present discussion, we will restrict ourselves to the deformation potential framework [31-34], which is expected to give the main contribution for exciton-phonon scattering in TMDs. Within this framework, the carrier-phonon coupling element $\left(g_{\mathbf{q}, \lambda}\right)$ is evaluated at the bottom/top of the conduction/valence band and is thus independent of the carrier momentum [31,32]; its expression reads

$$
g_{\mathbf{q}, \lambda}=\sqrt{\frac{\hbar}{2 \rho A \omega_{\mathbf{q}, \lambda}}} M_{\mathbf{q}, \lambda},
$$

where $\rho$ is the mass density of the monolayer, $A$ its area, $\omega_{\mathbf{q}, \lambda}$ is the energy of a phonon with momentum $\mathbf{q}$ belonging to the mode $\lambda$, and $M_{\mathbf{q}, \lambda}$ is the coupling matrix element. To describe intravalley scattering due to acoustic phonons, we assume a linear dispersion relation and $M_{\mathbf{q}, \lambda}=\Xi_{\lambda} q$, where the deformation potential $\Xi_{\lambda}$ is obtained from first-principles calculations. To describe intravalley scattering due to optical phonons and all intervalley scattering processes, we consider the dispersion relations of the involved phonons to be constant and the carrier-phonon matrix element to be given by the zero-order deformation potential $M_{\mathbf{q}, \lambda}=D_{\lambda}^{0}$. The values of the deformation potential are obtained from DFT calculations, like the ones of Jin et al. [34]. These values correspond to effective parameters capable of accurately describing transport properties. The acoustic deformation potentials contain the contributions of longitudinal acoustic and transverse acoustic phonons, while the optical ones are composed of the longitudinal optical, transverse optical, and $\mathrm{A}_{1}$ branches.

The Hamiltonian describing the exciton-phonon interaction can be obtained from the usual carrier-phonon Hamiltonian by quantizing it in terms of exciton operators, and it reads [35]

$$
\begin{aligned}
H_{\text {int }}= & \sum_{\mathbf{q}, \lambda} \sum_{\mathbf{Q} v \mu} \sum_{j \in \mathrm{BZ}}\left(a_{-j,-\mathbf{q}, \lambda}^{\dagger}+a_{j, \mathbf{q}, \lambda}\right) \\
\times & \times g_{\mathbf{q}}^{(e), \xi_{h},\left(\xi_{e}+j\right), \mu, v ; \lambda} b_{\mathbf{Q}+\mathbf{q}^{v}}^{\xi_{h},\left(\xi_{e}+j\right) \dagger} b_{\mathbf{Q} \mu}^{\xi_{h}, \xi_{e}} \\
& \left.-g_{\mathbf{q}}^{(h),\left(\xi_{h}-j\right), \xi_{e}, \mu, v ; \lambda} b_{\mathbf{Q}+\mathbf{q} v}^{\left(\xi_{h}+j\right), \xi_{e} \dagger} b_{\mathbf{Q} \mu}^{\xi_{h}, \xi_{e}}\right] .
\end{aligned}
$$


The first part of this Hamiltonian describes events where the exciton is scattered via scattering of the electron, while the second characterizes the processes with scattering of holes. In a more detailed way, the first term describes the scattering of the state $\left|\xi_{e}, \xi_{h}, \mu, \mathbf{Q}\right\rangle$ to the state $\left|\xi_{e}+j, \xi_{h}, v, \mathbf{Q}+\mathbf{q}\right\rangle$ due to the absorption/emission of a phonon of the mode $\lambda$ with momentum $-\mathbf{q} / \mathbf{q}$ measured relative to the point $-j / j$ of the Brillouin zone. A similar interpretation can be applied to the second term. The two $g_{\mathbf{q}}^{(\ldots)}$ characterize the exciton-phonon coupling strength, and their relation with the individual carrier-phonon coupling is given in Eq. (13). Note that this Hamiltonian describes both intravalley (setting $j=$ $\Gamma)$ and intervalley $(j \neq \Gamma)$ scattering events.

\section{Intravalley contribution}

Let us now focus on the particular case of intravalley scattering. Considering only the term with $j=\Gamma$ in $H_{\text {int }}$, we write

$$
H_{\mathrm{int}}^{\mathrm{intra}}=\sum_{\mathbf{q}, \lambda} \sum_{\mathbf{Q} v \mu} g_{\mathbf{q}}^{\mathrm{intra} ; \lambda}\left(a_{-\mathbf{q}, \lambda}^{\dagger}+a_{\mathbf{q}, \lambda}\right) b_{\mathbf{Q}+\mathbf{q} v}^{\dagger} b_{\mathbf{Q} \mu},
$$

where

$$
\begin{aligned}
g_{\mathbf{q}}^{\text {intra; } ;}= & g_{\mathbf{q}}^{(e), \mu, v ; \lambda}-g_{\mathbf{q}}^{(h), \mu, v ; \lambda} \\
= & \frac{1}{A} g_{\mathbf{q}, \lambda}^{(e)} \sum_{\mathbf{k}} \psi_{\mu}^{*}(\mathbf{k}+\beta \mathbf{q}) \psi_{\nu}(\mathbf{k}) \\
& -\frac{1}{A} g_{\mathbf{q}, \lambda}^{(h)} \sum_{\mathbf{k}} \psi_{\mu}^{*}(\mathbf{k}) \psi_{\nu}(\mathbf{k}+\alpha \mathbf{q}),
\end{aligned}
$$

with $\alpha=m_{e} /\left(m_{e}+m_{h}\right)$ and $\beta=m_{h} /\left(m_{e}+m_{h}\right)$. Note that, for simplicity, we omitted the indexes $\xi_{e}$ and $\xi_{h}$ since we consider that both carriers are in the $\mathrm{K}$ valley before and after the interaction takes place (the momenta of both carriers are measured relative to this point, while the momentum of the phonons is measured relative to the center of the respective Brillouin zone).

To compute the intravalley scattering contribution to the linewidth, we once again turn to Fermi's golden rule, this time with the interaction Hamiltonian of Eq. (12). Noting that, by definition, the transferred momentum in intravalley scattering processes is small, we take the limit of vanishing $\mathbf{q}$ in Eq. (13). Doing so and using the orthogonality of the exciton wave functions, we obtain

$$
g_{\mathbf{q}}^{\text {intra } ; \lambda} \approx\left[g_{\mathbf{q}, \lambda}^{(e)}-g_{\mathbf{q}, \lambda}^{(h)}\right] \delta_{\mu, \nu}
$$

This is in line with the idea that intravalley scattering should not induce transition between exciton states (e.g., $1 s \rightarrow 2 s$ ) since the energy required to originate such transitions far exceeds the energy phonons carry. We further note that, considering the exciton to be initially in the $1 s$ state with $\mathbf{Q}=$ 0 , only processes involving scattering through absorption of phonons are possible due to conservation of energy.

Applying Fermi's golden rule, we find

$$
\gamma_{\text {intra }}=2 \pi \sum_{\lambda, \mathbf{q}}\left|g_{\mathbf{q}, \lambda}^{(e)}-g_{\mathbf{q}, \lambda}^{(h)}\right|^{2} n\left(\omega_{\mathbf{q}, \lambda}\right) \delta\left(\frac{\hbar^{2} \mathbf{q}^{2}}{2 M}-\hbar \omega_{\mathbf{q}, \lambda}\right)
$$

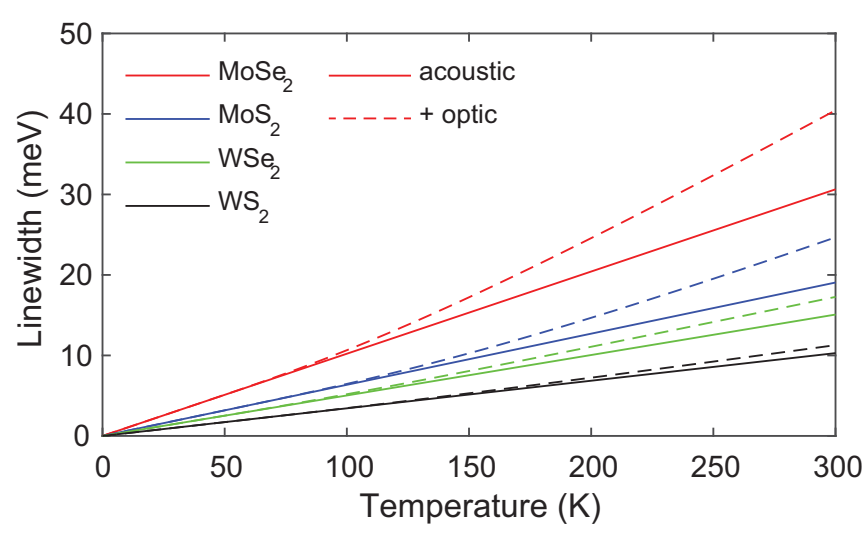

FIG. 3. Intravalley scattering induced linewidth for four different transition metal dichalcogenides (TMDs). The solid lines represent the contribution from acoustic phonons, and the dashed lines represent the combined contribution of acoustic and optical phonons. The parameters of Ref. [34] were used.

which gives the contributions

$$
\gamma_{\text {intra, ac }} \approx \frac{\left|\Xi^{(e)}-\Xi^{(h)}\right|^{2}}{\rho v_{\mathrm{ac}}^{2}} \frac{M}{\hbar^{2}} k_{B} T
$$

and

$$
\gamma_{\text {intra }, \text { op }}=M \frac{\left|D^{0,(e)}-D^{0,(h)}\right|^{2}}{2 \rho \hbar \omega_{\mathrm{op}}} n\left(\hbar \omega_{\mathrm{op}}\right),
$$

for the intravalley process due both to acoustic (ac) and optical (op) phonons. Here, $\Xi^{(e / h)}$ and $D^{0,(e / h)}$ are the first-order acoustic deformation potential and the zero-order optical deformation potential for electron/hole-phonon intravalley scattering, $n(\hbar \omega)$ is the Bose-Einstein distribution, $v_{\mathrm{ac}}$ is the speed of sound in the TMD, $\hbar \omega_{\text {op }}$ is the optical phonon energy, $M=m_{e}+m_{h}, \rho$ is the mass density of the monolayer [36], and $k_{B}$ is the Boltzmann's constant. As expected, we observe that the intravalley linewidth from acoustic phonons increases approximately linearly with temperature [33,34]. The contribution from optical phonons presents the same temperature dependence as the Bose-Einstein distribution function. From the DFT calculations of Ref. [34], one finds that the values of the deformation potential make scattering with acoustic phonons the dominant mechanism of the two, and thus, it is expected that the total intravalley scattering linewidth increases linearly with temperature with a small exponential correction. Also, we note that both contributions are insensitive to dielectric screening from the environment, which is a consequence of the vanishing transferred momentum approximation.

In Fig. 3, we depict the intravalley linewidth as a function of temperature for $\mathrm{MoS}_{2}, \mathrm{MoSe}_{2}, \mathrm{WS}_{2}$, and $\mathrm{WSe}_{2}$. We observe that this linewidth takes higher values for molybdenum (Mo)-based TMDs than for tungsten (W)-based ones because of the higher effective masses and deformation potentials of the former. Moreover, TMDs with the selenium (Se) are associated with larger broadenings than the ones with sulfur $(\mathrm{S})$; this is due to the higher values of $\rho v_{\mathrm{ac}}$ and $\hbar \omega_{\mathrm{op}}$ suppressing $\gamma_{\text {intra, ac }}$ and $\gamma_{\text {intra, op }}$, respectively, in TMDs with sulfur. 


\section{Intervalley contribution}

Now let us consider the contribution from intervalley scattering to the temperature dependence of the linewidth. As before, we assume that the exciton remains in the $1 s$ state before and after the scattering event. Moreover, for simplicity, we will consider that only the electron is scattered, going from the $K$ valley to other points of the Brillouin zone $(\Lambda$ and $\mathrm{K}^{\prime}$ valleys), while the hole remains in the $\mathrm{K}$ valley. This approximation is not expected to have a significant impact on the final result since the latter is a less efficient scattering process due to the smaller values of the deformation potentials [34].

The efficiency of intervalley scattering is directly related to the electronic band structure and the relative position of the exciton energy levels in different points of the Brillouin zone (cf., Fig. 2). In molybdenum-based TMDs, where the lowestlying exciton state is located at the $\mathrm{K}$ valley, we do not expect intervalley processes to play a significant role since the energetically more favorable scattering event $\mathrm{K} \rightarrow \mathrm{K}^{\prime}$ is rather inefficient. However, the situation is significantly different in tungsten-based TMDs, where the conduction band presents a satellite minimum halfway along the path from the vertices of the Brillouin zone to its center, the $\Lambda$ valley. In this region, the effective masses are larger than in the $\mathrm{K}$ valley, leading to more tightly bound excitons. As a consequence, in these TMDs, a momentum dark state at the $\Lambda$ valley appears below the optically active exciton. Since scattering events of the type $\mathrm{K} \rightarrow \Lambda$ are energetically favorable and the required momentum transfer is half of that required in $\mathrm{K} \rightarrow \mathrm{K}^{\prime}$ processes, it is expected that intervalley scattering offers a significant contribution to the total linewidth in tungsten-based TMDs.

To compute the intervalley linewidth, we return to Fermi's golden rule. Considering the aforementioned assumptions regarding the relevant scattering possibilities, we write the interaction Hamiltonian as

$$
\begin{aligned}
H_{\mathrm{int}}^{\mathrm{inter}}= & \sum_{\mathbf{q}, \lambda} \sum_{\mathbf{Q} v \mu} \sum_{j \in \mathrm{BZ}}\left(a_{-j,-\mathbf{q}, \lambda}^{\dagger}+a_{j, \mathbf{q}, \lambda}\right) \\
& \times g_{\mathbf{q}}^{(e), \xi_{h},\left(\xi_{e}+j\right), \mu, \nu ; \lambda} b_{\mathbf{Q}+\mathbf{q} v}^{\xi_{h},\left(\xi_{e}+j\right) \dagger} b_{\mathbf{Q} \mu}^{\xi_{h}, \xi_{e}} .
\end{aligned}
$$

Just as in the case of intravalley scattering events, the excitonphonon coupling is given by the product of the carrier-phonon coupling with a sum in momentum space of the wave functions of the initial and final exciton states, which are now located in different points of the Brillouin zone due to the nature of intervalley scattering. Explicitly,

$$
\begin{aligned}
g_{\mathbf{q}}^{(e), \xi_{h},\left(\xi_{e}+j\right), \mu, v ; \lambda}= & \frac{1}{A} \sum_{\mathbf{k}} g_{\mathbf{q}, \lambda}^{(e)} \psi_{\xi_{h},\left(\xi_{e}+j\right), \mu}^{*} \\
& \times\left[\mathbf{k}+\beta_{\xi_{h},\left(\xi_{e}+j\right)} \mathbf{q}\right] \psi_{\xi_{h}, \xi_{e}, v}(\mathbf{k}),
\end{aligned}
$$

with $\beta_{\xi_{h},\left(\xi_{e}+j\right)}=m_{h}^{\xi_{h}} / m_{e}^{\xi_{e}+j}+m_{h}^{\xi_{h}}$

Since this time around the exciton wave functions refer to different points of the Brillouin zone, this sum cannot be simply approximated to a Kronecker $\delta$ and needs to be explicitly computed. Because we have mapped the problem of both bright and dark excitons to the Wannier equation, which can be solved with a simple variational Ansatz, the sum in momentum space can still be computed analytically (see Appendix D). Thus, the nonradiative decay rate associated with the intervalley scattering of an electron from the $\mathrm{K}$ to the $\xi_{f}$ valley, with the hole remaining in the $\mathrm{K}$ valley (scattering of a $\mathrm{KK}$ exciton to a $\mathrm{K} \xi_{f}$ one), reads

$$
\begin{aligned}
\gamma_{\text {inter }, \mathrm{K} \xi_{f}} & \approx \sum_{\lambda, \pm} w \frac{\left|D_{\mathrm{K} \rightarrow \xi_{f}}^{0, \lambda,(e)}\right|^{2}}{\rho \hbar \omega_{\mathbf{j}, \lambda}} \frac{8 M_{\xi_{f}} a_{\mathrm{K}}^{2} a_{\xi_{f}}^{2}\left(a_{\mathrm{K}}+a_{\xi_{f}}\right)^{2}}{\left[\left(a_{\mathrm{K}}+a_{\xi_{f}}\right)^{2}+\beta_{\xi_{f}}^{2} a_{\mathrm{K}}^{2} a_{\xi_{f}}^{2} \mathbf{j}^{2}\right]^{3}} \\
& \times\left[\frac{1}{2} \pm \frac{1}{2}+n\left(\hbar \omega_{\mathbf{j}, \lambda}\right)\right] \Theta\left(-\Delta_{\mathrm{K} \xi_{f}} \mp \hbar \omega_{\mathbf{j}, \lambda}\right),
\end{aligned}
$$

where the sums are over the phonon modes $\lambda$ (acoustic and optical) and the emission/absorption $(+/-)$ of phonons, and $w$ is the degeneracy factor of the $\mathrm{K} \rightarrow \xi_{f}$ scattering event; $\mathbf{j}$ is the momentum given by $\mathrm{K}-\xi_{f} ; M_{\xi_{f}}=m_{e, \xi_{f}}+m_{h, \mathrm{~K}}$ is the translational mass of the $\mathrm{K} \xi_{f}$ exciton; and $\beta_{\xi_{f}}=m_{h, \mathrm{~K}} / M_{\xi_{f}}$. The Heaviside function $\Theta(x)$ sets the threshold for energetically allowed scattering processes, with $\Delta_{\mathrm{K} \xi_{f}}=\Delta_{\mathrm{K} \xi_{f}}^{\mathrm{CB}}+$ $\Delta_{\mathrm{K} \xi_{f}}^{\text {binding }}$ the energy difference of the conduction band edge at the $\mathrm{K}$ and $\xi_{f}$ valleys $\left(\Delta_{\mathrm{K} \xi_{f}}^{\mathrm{CB}}\right.$ ) plus the difference of the binding energy of the KK and $\mathrm{K} \xi_{f}$ excitons ( $\Delta_{\mathrm{K} \xi_{f}}^{\text {binding }}$ ), and $a_{\xi_{f}}\left(a_{\mathrm{K}}\right)$ is the variational parameter associated with the wave function of the $\mathrm{K} \xi_{f}(\mathrm{KK})$ exciton. The dependence of the variational parameters with $\mu_{\xi_{e}, \xi_{h}}, \kappa$, and $r_{0}$ is given by Eq. (7).

The appearance of the transferred momentum $\mathbf{j}$ in the denominator of Eq. (19) contributes to the suppression of intervalley processes such as $\mathrm{K} \rightarrow \mathrm{K}^{\prime}$ when compared with $\mathrm{K} \rightarrow \Lambda$. This is further enhanced by the deformation potential $D_{\mathrm{K} \rightarrow \xi_{f}}^{0, \lambda,(e)}$, which is approximately seven times larger in $\mathrm{K} \rightarrow \Lambda$ processes than in $\mathrm{K} \rightarrow \mathrm{K}^{\prime}$. Considering that, in Eq. (19), $\gamma_{\text {inter }}$ is proportional to the square of the deformation potential, it is clear that the contribution from $\mathrm{K} \rightarrow \Lambda$ is by far the dominant one. Moreover, transitions involving acoustic phonons have larger deformation potentials and thus are more relevant than the ones assisted by optical phonons. If $\Delta_{\mathrm{K} \xi_{f}}<0$, which is more likely to occur in tungsten-based TMDs due to the structure of its conduction band (cf., Fig. 2), then intervalley scattering is highly favorable and can occur via absorption or emission of phonons. On the other hand, if $\Delta_{\mathrm{K} \xi_{f}}>0$, then only absorption scattering channels are available, and even those may be suppressed depending on how $\Delta_{\mathrm{K} \xi_{f}}$ compares with the phonon energies (which range up to $\sim 50 \mathrm{meV}$ ). Contrary to intravalley scattering, the contribution from intervalley processes depends on the dielectric screening from the environment, owing to its dependence on the variational parameter $a$. Analyzing Eq. (19) combined with Eq. (7), we predict a decrease of linewidth with increasing dielectric screening roughly following a power law of the sixth order. Furthermore, as the dielectric screening increases, the binding energies decrease, and the $\mathrm{K} \Lambda$ excitons get closer to the optically bright one, leading to less energetically favorable intervalley processes, which results in the suppression of scattering channels and consequent decrease of the linewidth. This effect is depicted in Fig. 4, where we observe a sudden decrease in the nonradiative linewidth due to the suppression of the emission scattering channels. For higher values of the substrate dielectric constant, even the absorption scattering channels could be suppressed; however, for such values of screening, the intervalley linewidth becomes almost 

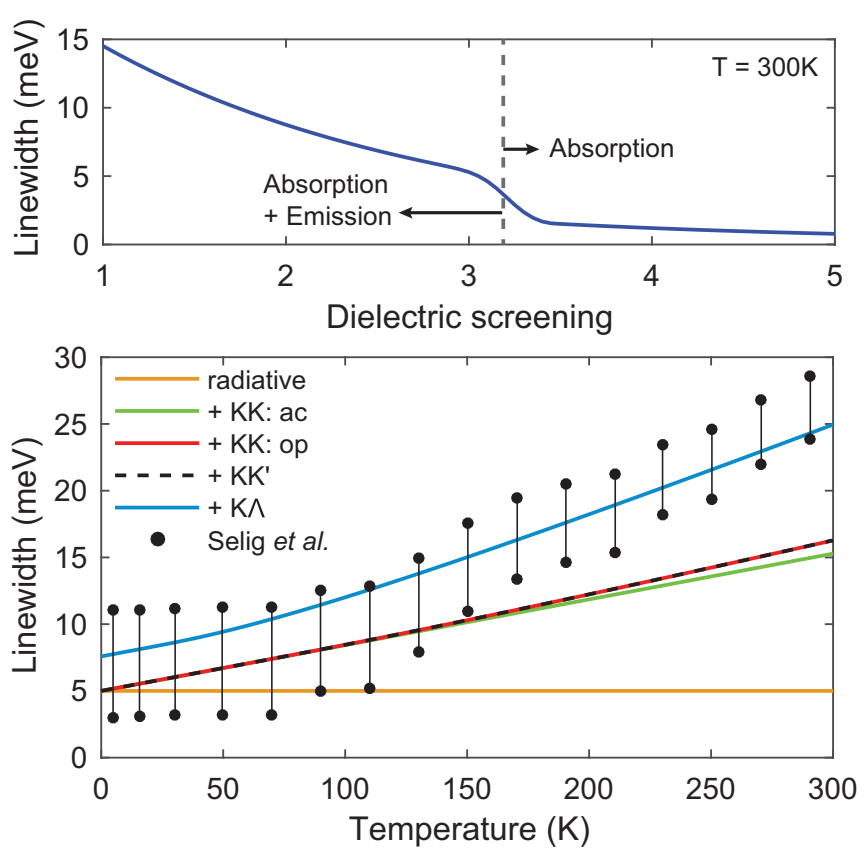

FIG. 4. (Top) Nonradiative linewidth as a function of the dielectric screening at $T=300 \mathrm{~K}$. (Bottom) Different contributions to the total linewidth of $\mathrm{WS}_{2}$ on quartz as a function of temperature. Contributions labeled by KK refer to intravalley scattering, while the others refer to intervalley contributions. The experimental points were taken from Ref. [11]. The parameters of Refs. [5,34] were used. For the intervalley contribution, we considered $m_{e}^{\mathrm{K}}=0.27 m_{0}$, $m_{h}^{\mathrm{K}}=0.36 m_{0}, m_{e}^{\mathrm{K}^{\prime}}=0.36 m_{0}, m_{e}^{\Lambda}=0.36 m_{0}, r_{0}=40 \AA, \hbar \omega_{\mathrm{ac}}=$ $16 \mathrm{meV}, \hbar \omega_{\mathrm{op}}=44 \mathrm{meV}, D_{0, \text { ac }}^{(e), \mathrm{K} \rightarrow \Lambda}=7.4 \times 10^{8} \mathrm{eV} / \mathrm{cm}, D_{0, \mathrm{op}}^{(e), \mathrm{K} \rightarrow \Lambda}=$ $0.9 \times 10^{8} \mathrm{eV} / \mathrm{cm}, D_{0, \text { ac }}^{(e), \mathrm{K} \rightarrow \mathrm{K}^{\prime}}=1.2 \times 10^{8} \mathrm{eV} / \mathrm{cm}$, and $D_{0, \mathrm{op}}^{(e), \mathrm{K} \rightarrow \mathrm{K}^{\prime}}=$ $1.1 \times 10^{8} \mathrm{eV} / \mathrm{cm}$. For the intravalley part, we used $\rho=4.32 \times$ $10^{-7} \mathrm{~g} / \mathrm{cm}^{2}, v_{\mathrm{ac}}=4.3 \times 10^{5} \mathrm{~cm} / \mathrm{s}, \Xi^{(e)}=3.2 \mathrm{eV}, \Xi^{(h)}=-1.7 \mathrm{eV}$, $D_{0}^{(e)}=3.1 \times 10^{8} \mathrm{eV} / \mathrm{cm}$, and $D_{0}^{(h)}=-2.3 \times 10^{8}$. For the radiative contribution, we used $\hbar v_{F}=3.34 \mathrm{eVA}, E_{g}=2.88 \mathrm{eV}$, and $E_{g}^{\tau, s_{z}}=$ $1.61 \mathrm{eV}$.

insignificant even before the suppression of the channel. Band gap engineering and changes in the effective masses may also be used to enhance (or suppress) different scattering channels.

\section{RESULTS: APPLICATION TO $\mathrm{WS}_{2}$}

Now that all the necessary analytical expressions were derived, we study the specific case of the variation of the $1 \mathrm{~s}$ exciton linewidth in $\mathrm{WS}_{2}$ with temperature and compare our theoretical prediction with the experimental data of Ref. [11]; in Fig. 4, a good agreement between theory and experiment is seen. We note that the total linewidth is composed of a constant radiative term of $\sim 5 \mathrm{meV}$ combined with the almost linear contribution from intravalley scattering (cf., Fig. 3) and an exponentially increasing intervalley term. These contributions amount to a total linewidth at room temperature of $\sim 25 \mathrm{meV}$. Moreover, the intervalley contributions stem almost entirely from $\mathrm{K} \rightarrow \Lambda$ events as a consequence of the low efficiency of the $\mathrm{K} \rightarrow \mathrm{K}^{\prime}$ scattering processes (something similar would happen with the $\mathrm{K} \rightarrow \Gamma$ hole scattering contribution). In the low temperature region, we observe that, contrary to the intravalley linewidth, the intervalley term is finite due to the efficient process of scattering with phonon emission. As the temperature increases, so do the intravalley and intervalley contributions, presenting similar magnitudes at room temperature. A similar calculation for $\mathrm{WSe}_{2}$ yields an identical result, with a slightly larger linewidth, as a consequence of the more favorable intravalley scattering processes (cf., Fig. 3). For $\mathrm{MoS}_{2}$ and $\mathrm{MoSe}_{2}$, where intervalley processes are less efficient, the total linewidth is basically given by the radiative and intravalley contributions.

\section{FINAL REMARKS}

In summary, solving the Wannier equation with a variational Ansatz, we obtained the binding energies and wave functions of both bright and momentum dark excitons. Then employing Fermi's golden rule, we derived analytical expressions describing the radiative, intravalley, and intervalley contributions to the total linewidth of the lowest-lying exciton resonance in different TMDs at finite temperature. Our theoretical prediction is in good agreement with experimental data. The derived expressions combined with parameters computed from DFT calculations allow for easily accessible estimates of the different contributions for the linewidth at any temperature value and give insight on how these quantities depend on the material and environment parameters.

\section{ACKNOWLEDGMENTS}

N.M.R.P. acknowledges support by the Portuguese Foundation for Science and Technology (FCT) in the framework of the Strategic Funding UIDB/04650/2020. J.C.G.H. acknowledges the Center of Physics for a grant funded by the UIDB/04650/2020 strategic project and POCI-01-0145FEDER-028887. N.M.R.P. acknowledges support from the European Commission through the project "Graphene-Driven Revolutions in ICT and Beyond" (Ref. No. 881603, CORE 3), COMPETE 2020, PORTUGAL 2020, FEDER, and the FCT through projects POCI-01-0145-FEDER-028114 and PTDC/NAN-OPT/29265/2017. N.A.M. is a VILLUM Investigator supported by VILLUM FONDEN (Grant No. 16498). The Center for Nanostructured Graphene is sponsored by the Danish National Research Foundation (Project No. DNRF103).

\section{APPENDIX A: DETAILS ON THE WANNIER EQUATION}

In this Appendix, we outline the necessary steps to obtain the Wannier equation in its differential form as presented in the main text.

To obtain the Wannier equation, we start by defining the exciton creation operator using electronic operators:

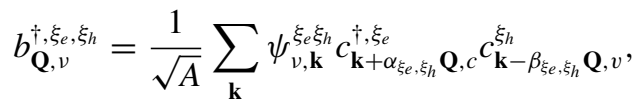

where $A$ is the area of the monolayer; $\psi_{\nu, \mathbf{k}}^{\xi_{e}, \xi_{h}}$ is the Fourier transform of an exciton with quantum numbers denoted by $v$, with the electron located in the $\xi_{e}$ point of the Brillouin zone and the hole in the $\xi_{h}$ point; and the relative momentum $\mathbf{k}$ is defined with respect to the $\xi_{e}$ and $\xi_{h}$ points. The operator $c_{\mathbf{k}+\alpha_{\xi e, \xi_{h}}^{\dagger}, \mathbf{Q}, c}$ creates an electron in the conduction band in 
the vicinity of point $\xi_{e}$ of the Brillouin zone, with momentum $\mathbf{k}+\alpha_{\xi_{e}, \xi_{h}} \mathbf{Q}$ measured relative to $\xi_{e}$ (a similar definition applies to $\left.c_{\mathbf{k}-\beta_{\xi_{e}, \xi_{h}} \mathbf{Q}, v}\right)$. Also, $\alpha_{\xi_{e}, \xi_{h}}=m_{e}^{\xi_{e}} /\left(m_{e}^{\xi_{e}}+m_{h}^{\xi_{h}}\right)$, and $\beta_{\xi_{e}, \xi_{h}}=m_{h}^{\xi_{e}} /\left(m_{e}^{\xi_{e}}+m_{h}^{\xi_{h}}\right)$. Hence, $b_{\mathbf{Q}, v}^{\dagger, \xi_{e}, \xi_{h}}$ corresponds to the creation of an exciton with quantum numbers $v$ and center of mass momentum $\mathbf{Q}$ measured relative to the high-symmetry points $\xi_{e}$ and $\xi_{h}$. The total momentum of the exciton reads $\mathbf{Q}_{\text {total }}=\xi_{e}-\xi_{h}+\mathbf{Q}$. When this operator acts on the excitonic ground state (a full valence band and an empty conduction band), we find

$$
b_{\mathbf{Q}, v}^{\dagger, \xi_{e}, \xi_{h}}|G S\rangle=\left|\xi_{e}, \xi_{h}, v, \mathbf{Q}\right\rangle .
$$

The energy of such a state reads

$$
E_{\mathbf{Q}, v}^{\xi_{e}, \xi_{h}}=E_{g}^{\xi_{e}, \xi_{h}}+E_{\nu}^{\xi_{e}, \xi_{h}}+\frac{\hbar^{2} \mathbf{Q}^{2}}{2 M_{\xi_{e}, \xi_{h}}},
$$

with $E_{g}^{\xi_{e}, \xi_{h}}$ the band gap between the $\xi_{e}$ and $\xi_{h}$ points, $E_{\nu}^{\xi_{e}, \xi_{h}}$ the exciton binding energy, and $\hbar^{2} \mathbf{Q}^{2} / 2 M_{\xi_{e}, \xi_{h}}$ its center of mass kinetic energy. This last term originates the parabolic dispersion near the high-symmetry point $\xi_{e}-\xi_{h}$ in the exciton Brillouin zone. Note that indexes such as $\xi_{e}$ refer to points in the Brillouin zone and as a consequence can be seen as momenta measured relative to the $\Gamma$ point.

The Hamiltonian describing electron-electron interactions is composed of a kinetic and a potential term and can be quantized in terms of electron operators as

$$
\hat{H}_{0}=\sum_{\mathbf{k}, \lambda, \xi} E_{\mathbf{k}, \lambda}^{\xi} c_{\mathbf{k}, \lambda}^{\dagger, \xi} c_{\mathbf{k}, \lambda}^{\xi}
$$

The potential term reads

$$
\begin{aligned}
\hat{H}_{\text {int }}= & \frac{1}{2 A} \sum_{\{\lambda, \xi\}\}} \sum_{\mathbf{q}, \mathbf{k}_{3}, \mathbf{k}_{4}} V(\mathbf{q}) F_{\lambda_{1}, \lambda_{2}, \lambda_{3}, \lambda_{4}}^{\xi_{1}, \xi_{2}, \xi_{3}, \xi_{4}}\left(\mathbf{k}_{3}, \mathbf{k}_{4}, \mathbf{q}\right) \\
& \times c_{\mathbf{k}_{4}+\mathbf{q} \lambda_{1}}^{\dagger, \xi_{1}} c_{\mathbf{k}_{3}-\mathbf{q} \lambda_{2}}^{\dagger, \xi_{2}} c_{\mathbf{k}_{3} \lambda_{3}}^{\xi_{3}} c_{\mathbf{k}_{4} \lambda_{4}}^{\xi_{4}},
\end{aligned}
$$

where $V(\mathbf{q})$ is the Fourier transform of the interaction potential, and

$$
F_{\lambda_{1}, \lambda_{2}, \lambda_{3}, \lambda_{4}}^{\xi_{1}, \xi_{2}, \xi_{3}, \xi_{4}}\left(\mathbf{k}_{3}, \mathbf{k}_{4}, \mathbf{q}\right)=u_{\mathbf{k}_{4}+\mathbf{q} \lambda_{1}}^{\dagger, \xi_{1}} u_{\mathbf{k}_{3}-\mathbf{q} \lambda_{2}}^{\dagger, \xi_{2}} u_{\mathbf{k}_{3} \lambda_{3}}^{\xi_{3}} u_{\mathbf{k}_{4} \lambda_{4}}^{\xi_{4}},
$$

with $u_{\mathbf{k}}^{\xi}$ the band spinor of the $\xi$ point evaluated at $\mathbf{k}$. Note that the sum over the transferred momentum in Eq. (A5) excludes the term $\mathbf{q}=0$ since this term cancels with the background contribution in the jellium model.

To obtain the equation that the defines the wave functions $\psi_{\nu, \mathbf{k}}^{\xi_{e} \xi_{h}}$ and the associated binding energies, we state that the total Hamiltonian is diagonal in the exciton operator representation, that is,

$$
\hat{H}=\sum_{\mathbf{Q}, v, \xi_{e}, \xi_{h}} E_{\mathbf{Q}, v}^{\xi_{e}, \xi_{h}} b_{\mathbf{Q}, v}^{\dagger, \xi_{e}, \xi_{h}} b_{\mathbf{Q}, v}^{\xi_{e}, \xi_{h}} .
$$

This is in principle always possible, given that the exciton wave function is correctly chosen. Then we compute the commutator $\left(\hat{H}, b_{\mathbf{Q}, v}^{\dagger, \xi_{e}, \xi_{h}}\right)$ using the exciton and the electron representation of the Hamiltonian and demand both results to be equal. Although we may keep the center of mass momentum $\mathbf{Q}$ finite throughout the calculation, in the end, we set it equal to zero since our goal is to compute the exciton binding energy, and as a result, we are interested in the bottom of the parabolic energy dispersion relation. In the end, we arrive at an integral equation in momentum space, which reads

$$
\begin{aligned}
E_{\mathbf{0}, v}^{\xi_{e}, \xi_{h}} \psi_{v, \mathbf{k}}^{\xi_{e} \xi_{h}}= & \psi_{\nu, \mathbf{k}}^{\xi_{e} \xi_{h}}\left(E_{\mathbf{k}, c}^{\xi_{e}}-E_{\mathbf{k}, v}^{\xi_{h}}\right) \\
& -\frac{1}{A} \sum_{\mathbf{p}} \psi_{v, \mathbf{p}}^{\xi_{e} \xi_{h}} V(\mathbf{k}-\mathbf{p}) F_{c, v, v, c}^{\xi_{e}, \xi_{h}, \xi_{h}, \xi_{e}}(\mathbf{k}, \mathbf{p}, \mathbf{k}-\mathbf{p}) .
\end{aligned}
$$

The term on the left-hand side of the equation corresponds to the product of the exciton energy $\left(E_{\mathbf{0}, v}^{\xi_{e}, \xi_{h}}\right)$ and its wave function in momentum space $\left(\psi_{v, \mathbf{k}}^{\xi_{e} \xi_{h}}\right)$. On the right-hand side, the first term gives the energy difference of the electron and hole in the noninteracting limit, and the second term describes the electron-hole attractive interaction. Now two approximations are introduced: (i) the term $\left(E_{\mathbf{k}, c}^{\xi_{e}}-E_{\mathbf{k}, v}^{\xi_{h}}\right)$ is expanded to the second order in $\mathbf{k}$, yielding $E_{g}^{\xi_{g}, \xi_{h}}+\hbar^{2} \mathbf{k}^{2} / 2 \mu^{\xi_{e} \xi_{h}}$, with $\mu$ the electron-hole reduced mass (note that the term $E_{g}^{\xi_{e}, \xi_{h}}$ does not contribute to the binding energy); and (ii) the form factor $F_{c, v, v, c}^{\xi_{e}, \xi_{h}, \xi_{h}, \xi_{e}}(\mathbf{k}, \mathbf{p}, \mathbf{k}-\mathbf{p})$ is approximated to one, which is a good approximation in the $\mathrm{K} / \mathrm{K}^{\prime}$ valleys due to the large energy gap and identically true in the $\Lambda$ valley). With these two approximations, this integral equation in momentum space can be transformed into a differential one by means of a Fourier transform. Doing so, we arrive at the Wannier equation given in the main text:

$$
-\frac{\hbar^{2}}{2 \mu_{\xi_{e} \xi_{h}}} \nabla^{2} \psi_{v}^{\xi_{e} \xi_{h}}(\mathbf{r})+V_{\mathrm{RK}}(\mathbf{r}) \psi_{v}^{\xi_{e} \xi_{h}}(\mathbf{r})=E_{v}^{\xi_{e} \xi_{h}} \psi_{v}^{\xi_{e} \xi_{h}}(\mathbf{r}) .
$$

\section{APPENDIX B: TESTING THE VARIATIONAL APPROACH}

In the main text, we introduced the variational Ansatz

$$
\psi_{1 s}(\mathbf{r})=\sqrt{\frac{2}{\pi a^{2}}} e^{-r / a},
$$

to describe the wave function of the excitonic $1 s$ state. We also found that the variational parameter can be accurately described by the relation

$$
\frac{a}{a_{B}} \simeq \frac{\kappa}{\mu / m_{0}} \chi+\sqrt{\frac{r_{0} / a_{B}}{\mu / m_{0}}} .
$$

The binding energy can then be obtained from

$$
E_{1 s}=\int \psi_{1 s}^{*}(r) H \psi_{1 s}(r) d \mathbf{r},
$$

where $H$ is the Hamiltonian containing the kinetic energy and the RK potential. Analytically, this reads

$$
\begin{aligned}
E_{1 s}= & \frac{\hbar^{2}}{2 \mu a^{2}} \\
& +\frac{\alpha \hbar c}{r_{0}}\left[\frac{1-x}{1+x^{2}}-\frac{\operatorname{ArcCsch}(x)+\operatorname{ArcSinh}(x)}{\left(1+x^{2}\right)^{3 / 2}}\right],
\end{aligned}
$$

with $x=a \kappa /\left(2 r_{0}\right)$. In Table I, we present the binding energies for different excitons obtained using Eq. (B3) and by numerically solving the Wannier equation. An excellent agreement between the two approaches is seen when the two sets of data 
TABLE I. Binding energies (in meV) for the excitons depicted in Fig. 2, obtained with the variational Ansatz and by numerically solving the Wannier equation. The effective masses of Ref. [3] were used.

\begin{tabular}{|c|c|c|c|c|c|c|}
\hline & \multicolumn{2}{|c|}{ KK } & \multicolumn{2}{|c|}{$\mathrm{KK}^{\prime}$} & \multicolumn{2}{|c|}{$\mathrm{K} \Lambda$} \\
\hline & Var. & Num. & Var. & Num. & Var. & Num. \\
\hline $\mathrm{MoS}_{2}$ & -354 & -361 & -359 & -366 & -394 & -401 \\
\hline $\mathrm{MoSe}_{2}$ & -318 & -325 & -326 & -333 & -340 & -347 \\
\hline $\mathrm{WS}_{2}$ & -317 & -324 & -336 & -343 & -368 & -375 \\
\hline $\mathrm{WSe}_{2}$ & -289 & -295 & -307 & -314 & -325 & -331 \\
\hline
\end{tabular}

are compared, with a relative difference of $\sim 2 \%$. The quality of these results validates the use of the variational approach.

\section{APPENDIX C: DETAILS ON THE RADIATIVE LINEWIDTH}

Using Fermi's golden rule, we define the radiative linewidth of the exciton in the state $v$ as

$$
\begin{aligned}
\gamma_{\mathrm{rad}}=\frac{\hbar}{\tau_{v}}= & 2 \pi \sum_{\mathbf{q}}\left|\left\langle G S ; 1_{\mathbf{q}}|e \mathbf{E} \cdot \mathbf{r}| \nu ; \mathbf{Q}=0\right\rangle\right|^{2} \\
& \times \delta\left(E_{g}+E_{v}-\hbar \omega_{\mathbf{q}}\right) .
\end{aligned}
$$

To compute the matrix element, we start by writing the position operator $\mathbf{r}$ in the electronic basis as

$$
\begin{aligned}
\mathbf{r} & =\sum_{\mathbf{k}}\left\langle u_{\mathbf{k}, c}\left|i \nabla_{\mathbf{k}}\right| u_{\mathbf{k}, v}\right\rangle c_{\mathbf{k}, c}^{\dagger} c_{\mathbf{k}, v}+\text { H.c. } \\
& =i \sum_{\mathbf{k}} \xi_{\mathbf{k} c v} c_{\mathbf{k}, c}^{\dagger} c_{\mathbf{k}, v}+\text { H.c. }
\end{aligned}
$$

where $\nabla_{\mathbf{k}}$ is the gradient operator in momentum space; $c_{\mathbf{k}, v}$ $/ c_{\mathbf{k}, c}^{\dagger}$ is the operator that annihilates/creates an electron with momentum $\mathbf{k}$ in the valence/conduction band; $\left|u_{\mathbf{k}, v / c}\right\rangle$ is the periodic part of the Bloch function for the valence/conduction band and can be obtained as the eigenvector of the effective Hamiltonian (including spin-orbit coupling) in the vicinity of the $\mathrm{K}$ valley; and $\boldsymbol{\xi}_{\mathbf{k} c v}=\left\langle u_{\mathbf{k}, c}\left|\nabla_{\mathbf{k}}\right| u_{\mathbf{k}, v}\right\rangle$ is termed the Berry connection. The electric field is quantized in the usual way and reads

$$
\mathbf{E} \approx i \sum_{\mathbf{q}, \lambda} \sqrt{\frac{\hbar \omega_{\mathbf{q}}}{2 V \epsilon_{0}}}\left[\mathbf{e}_{\lambda} a_{\mathbf{q}, \lambda}(t)-\left(\mathbf{e}_{\lambda}\right)^{*} a_{\mathbf{q}, \lambda}^{\dagger}(t)\right],
$$

where the sum over $\lambda$ corresponds to a sum over the polarization of light, $\mathbf{e}_{\lambda}$ is a polarization vector, $V$ is the volume of the system, $\epsilon_{0}$ is the permittivity of vacuum, and $a_{\mathbf{q}, \lambda} / a_{\mathbf{q}, \lambda}^{\dagger}$ is the annihilation/creation operator of a photon with momentum $\mathbf{q}$ with polarization $\lambda$. Computing the matrix element that appears in Eq. (C1), we obtain

$$
\frac{1}{\tau_{v}}=\frac{4 \pi}{\hbar} \alpha\left(E_{g}+E_{v}\right)\left|\frac{1}{A} \sum_{\lambda, \mathbf{k}} \psi_{\nu}(\mathbf{k}) \xi_{\mathbf{k} c v}^{*} \cdot\left(\mathbf{e}_{\lambda}\right)^{*}\right|^{2},
$$

where $\alpha \sim 1 / 137$ is the fine structure constant. To compute the Berry connection, we approximate $\left|u_{\mathbf{k}, v / c}\right\rangle$ by its form near the top/bottom of the valence/conduction band, that is, we perform a $\mathbf{k} \cdot \mathbf{p}$ expansion around the respective highsymmetry point of the Brillouin zone. In the end, we find

$$
\frac{1}{\tau_{v}}=\frac{8 \pi}{\hbar} \alpha\left(E_{g}+E_{v}\right)\left(\frac{\hbar v_{F}}{E_{g}^{\tau, s_{z}}}\right)^{2} \psi_{\nu}^{2}(\mathbf{r}=0) .
$$

For a TMD in a dielectric medium with dielectric constant $\kappa$, we obtain

$$
\frac{\hbar}{\tau_{v}}=\gamma_{\mathrm{rad}}=\frac{8 \pi}{\kappa} \alpha\left(E_{g}+E_{v}\right)\left(\frac{\hbar v_{F}}{E_{g}^{\tau, s_{z}}}\right)^{2} \psi_{\nu}^{2}(\mathbf{r}=0) .
$$

This expression is the generalization of the result given in the main text since it is valid for an arbitrary state $v$.

\section{APPENDIX D: DETAILS ON THE INTERVALLEY LINEWIDTH}

For intervalley scattering, we consider the initial exciton state to have both electron and hole in the $\mathrm{K}$ valley and vanishing center of mass momentum (relative to this valley); its energy reads $E_{g}^{\mathrm{KK}}+E_{1 s}^{\mathrm{KK}}$. The final exciton state corresponds to an exciton where the hole is in the $\mathrm{K}$ valley and the electron in the vicinity of $\mathrm{K}+j$ (which we consider to be either the $\mathrm{K}^{\prime}$ or $\Lambda$ valleys); its energy reads $E_{g}^{\mathrm{K}(\mathrm{K}+j)}+E_{1 s}^{\mathrm{K}(\mathrm{K}+j)}+$ $\hbar^{2} \mathbf{q}^{2} / 2 M_{\mathrm{K}(\mathrm{K}+j)}$.

Using Fermi's golden rule, one finds

$$
\begin{aligned}
\gamma_{\text {inter }}= & 2 \pi \sum_{\lambda, \pm} \sum_{\mathbf{q}, j \neq \Gamma} w_{j}\left|g_{\mathbf{q}}^{\mathrm{K},(\mathrm{K}+j), 1 s, 1 s ; \lambda}\right|^{2} \\
& \times\left[\frac{1}{2} \pm \frac{1}{2}+n\left(\omega_{j, \mathbf{q}, \lambda}\right)\right] \\
& \times \delta\left[\frac{\hbar^{2} \mathbf{q}^{2}}{2 M_{\mathrm{K}(\mathrm{K}+j)}}+\Delta_{\mathrm{K} j} \pm \hbar \omega_{j, \mathbf{q}, \lambda}\right],
\end{aligned}
$$

where $w_{j}$ is the degeneracy factor of the $\mathrm{K} \rightarrow \mathrm{K}+j$ scattering event, and $\Delta_{\mathrm{K} j}=E_{g}^{\mathrm{K}(\mathrm{K}+j)}-E_{g}^{\mathrm{KK}}+E_{1 s}^{\mathrm{K}(\mathrm{K}+j)}-E_{1 s}^{\mathrm{KK}}$. Since the hole remains in the $\mathrm{K}$ valley. this is the same as $\Delta_{\mathrm{K} j}=\Delta_{\mathrm{K} j}^{\mathrm{CB}}+\Delta_{\mathrm{K} j}^{\text {binding }}$, that is, the energy difference between the bottom of the conduction band in the two valleys plus the difference in the binding energies. The coupling strength reads

$$
\begin{aligned}
g_{\mathbf{q}}^{\mathrm{K},(\mathrm{K}+j), 1 s, 1 s ; \lambda}= & \frac{1}{A} \sum_{\mathbf{k}} g_{\mathbf{q}}^{(e), \lambda} \psi_{\mathrm{K},(\mathrm{K}+j), 1 s}^{*} \\
& \times\left[\mathbf{k}+\beta_{\mathrm{K},(\mathrm{K}+j)} \mathbf{q}\right] \psi_{\mathrm{K}, \mathrm{K}, 1 s}(\mathbf{k}),
\end{aligned}
$$

where contrarily to the intravalley case, the valleys where the electron and hole sit must be explicitly stated. We note that the value of $g_{\mathbf{q}}^{(e), \lambda}$ is not the same as in the intravalley case and should be chosen according to the scattering event in question [e.g., the scattering $\mathrm{K} \rightarrow \mathrm{K}^{\prime}$ has a different value for $g_{\mathbf{q}}^{(e), \lambda}$ than the one used to describe the $\mathrm{K} \rightarrow \Gamma$ event]. This coupling element is associated with the process where a phonon from the vicinity of the $j$ point (e.g., K) of the phonon Brillouin zone is absorbed, and as a consequence, the electron leaves the $\mathrm{K}$ valley to the region near the $\mathrm{K}+j$ valley (e.g., $\mathrm{K}+\mathrm{K}=\Gamma$ ); the transferred momentum is $j+\mathbf{q}$. Introducing the Fourier 
representation of the wave functions, we find

$$
\begin{aligned}
g_{\mathbf{q}}^{\mathrm{K},(\mathrm{K}+j), 1 s, 1 s ; \lambda}= & g_{\mathbf{q}}^{(e), \lambda} \int \psi_{\mathrm{K},(\mathrm{K}+j), 1 s}^{*}(\mathbf{r}) \psi_{\mathrm{K}, \mathrm{K}, 1 s} \\
& \times(\mathbf{r}) \exp \left[i \beta_{\mathrm{K},(\mathrm{K}+j)} \mathbf{r} \cdot(j+\mathbf{q})\right] d \mathbf{r} .
\end{aligned}
$$

The exciton wave functions can be described with the single exponential variational Ansatz given in the main text. Contrary to the intravalley case, the complex exponential cannot be approximated to one due to the large transferred momentum. However, considering $|j| \gg|\mathbf{q}|$ and using the variational
Ansatz for the $1 s$ state to describe the wave functions, we can approximate this by

$$
g_{\mathbf{q}}^{\mathrm{K},(\mathrm{K}+j), 1 s, 1 s ; \lambda}=g_{\mathbf{q}}^{(e), \lambda} \frac{4 a_{1} a_{2}\left(a_{1}+a_{2}\right)}{\left[\left(a_{1}+a_{2}\right)^{2}+\left(a_{1} a_{2} \beta_{2}|j|\right)^{2}\right]^{3 / 2}},
$$

where to simplify the notation, we introduced the indexes 1 and 2, which correspond to the valley information of the initial and final states, respectively; that is, $1 \equiv\{\mathrm{K}, \mathrm{K}\}$ and $2 \equiv\{\mathrm{K}, \mathrm{K}+j\}$. Inserting this result into $\gamma_{\text {inter }}$ and recalling that, for intervalley processes, $g_{\mathbf{q}}^{(e)}$ is described by a zerothorder deformation potential, one recovers the result given in the main text.
[1] G. Berghäuser and E. Malic, Phys. Rev. B 89, 125309 (2014).

[2] G. Wang, A. Chernikov, M. M. Glazov, T. F. Heinz, X. Marie, T. Amand, and B. Urbaszek, Rev. Mod. Phys. 90, 021001 (2018).

[3] M. Koperski, M. R. Molas, A. Arora, K. Nogajewski, A. O. Slobodeniuk, C. Faugeras, and M. Potemski, Nanophotonics 6, 1289 (2017).

[4] D. Xiao, G.-B. Liu, W. Feng, X. Xu, and W. Yao, Phys. Rev. Lett. 108, 196802 (2012).

[5] A. Kormányos, G. Burkard, M. Gmitra, J. Fabian, V. Zólyomi, N. D. Drummond, and V. Fal'ko, 2D Mater. 2, 022001 (2015).

[6] E. Malic, M. Selig, M. Feierabend, S. Brem, D. Christiansen, F. Wendler, A. Knorr, and G. Berghäuser, Phys. Rev. Materials 2, 014002 (2018).

[7] X.-X. Zhang, Y. You, S. Y. F. Zhao, and T. F. Heinz, Phys. Rev. Lett. 115, 257403 (2015).

[8] H. Yu, M. Laurien, Z. Hu, and O. Rubel, Phys. Rev. B 100, 125413 (2019).

[9] G. Berghäuser, P. Steinleitner, P. Merkl, R. Huber, A. Knorr, and E. Malic, Phys. Rev. B 98, 020301(R) (2018).

[10] H. Dery and Y. Song, Phys. Rev. B 92, 125431 (2015).

[11] M. Selig, G. Berghäuser, A. Raja, P. Nagler, C. Schüller, T. F. Heinz, T. Korn, A. Chernikov, E. Malic, and A. Knorr, Nat. Commun. 7, 13279 (2016).

[12] C. Poellmann, P. Steinleitner, U. Leierseder, P. Nagler, G. Plechinger, M. Porer, R. Bratschitsch, C. Schüller, T. Korn, and R. Huber, Nat. Mater. 14, 889 (2015).

[13] P. Dey, J. Paul, Z. Wang, C. E. Stevens, C. Liu, A. H. Romero, J. Shan, D. J. Hilton, and D. Karaiskaj, Phys. Rev. Lett. 116, 127402 (2016).

[14] M. Feierabend, G. Berghäuser, A. Knorr, and E. Malic, Nat. Commun. 8, 14776 (2017).

[15] Q. Ma, G. Ren, K. Xu, and J. Z. Ou, Adv. Opt. Mater. 9, 2001313 (2021).

[16] T. Mueller and E. Malic, npj 2D Mater. Appl. 2, 29 (2018).

[17] K. F. Mak, D. Xiao, and J. Shan, Nat. Photonics 12, 451 (2018).

[18] R. Lv, J. A. Robinson, R. E. Schaak, D. Sun, Y. Sun, T. E. Mallouk, and M. Terrones, Acc. Chem. Res. 48, 56 (2015).
[19] F. Cadiz, E. Courtade, C. Robert, G. Wang, Y. Shen, H. Cai, T. Taniguchi, K. Watanabe, H. Carrere, D. Lagarde, M. Manca, T. Amand, P. Renucci, S. Tongay, X. Marie, and B. Urbaszek, Phys. Rev. X 7, 021026 (2017).

[20] N. S. Rytova, Moscow Univ. Phys. Bull. 22, 3 (1967).

[21] L. V. Keldysh, Sov. J. Exp. Theor. Phys. Lett. 29, 658 (1979).

[22] P. Cudazzo, I. V. Tokatly, and A. Rubio, Phys. Rev. B 84, 085406 (2011).

[23] H. Haug and S. W. Koch, Quantum Theory of the Optical and Electronic Properties of Semiconductors (World Scientific Publishing Company, Singapore, 2009).

[24] M. F. C. Martins Quintela and N. M. R. Peres, Eur. Phys. J. B 93, 222 (2020).

[25] T. G. Pedersen, Phys. Rev. B 94, 125424 (2016).

[26] T. Olsen, S. Latini, F. Rasmussen, and K. S. Thygesen, Phys. Rev. Lett. 116, 056401 (2016).

[27] T. Deilmann and K. S. Thygesen, 2D Mater. 6, 035003 (2019).

[28] M. Palummo, M. Bernardi, and J. C. Grossman, Nano Lett. 15, 2794 (2015).

[29] J. C. G. Henriques, G. Catarina, A. T. Costa, J. FernándezRossier, and N. M. R. Peres, Phys. Rev. B 101, 045408 (2020).

[30] G. Scuri, Y. Zhou, A. A. High, D. S. Wild, C. Shu, K. De Greve, L. A. Jauregui, T. Taniguchi, K. Watanabe, P. Kim, M. D. Lukin, and H. Park, Phys. Rev. Lett. 120, 037402 (2018).

[31] K. Kaasbjerg, K. S. Thygesen, and K. W. Jacobsen, Phys. Rev. B 85, 115317 (2012).

[32] K. Kaasbjerg, K. S. Thygesen, and A.-P. Jauho, Phys. Rev. B 87, 235312 (2013).

[33] X. Li, J. T. Mullen, Z. Jin, K. M. Borysenko, M. Buongiorno Nardelli, and K. W. Kim, Phys. Rev. B 87, 115418 (2013).

[34] Z. Jin, X. Li, J. T. Mullen, and K. W. Kim, Phys. Rev. B 90, 045422 (2014).

[35] M. Selig, Exciton-phonon coupling in monolayers of transition metal dichalcogenides, Ph.D. thesis, Technische Universitaet Berlin, Germany, 2018.

[36] S. Shree, M. Semina, C. Robert, B. Han, T. Amand, A. Balocchi, M. Manca, E. Courtade, X. Marie, T. Taniguchi, K. Watanabe, M. M. Glazov, and B. Urbaszek, Phys. Rev. B 98, 035302 (2018). 